\title{
Optimal achromatic wave retarders using two birefringent wave plates
}

\author{
Jose Luis Vilas, ${ }^{*}$ Luis Miguel Sanchez-Brea, and Eusebio Bernabeu \\ Optics Department, Applied Optics Complutense Group, Universidad Complutense de Madrid, \\ Facultad de Ciencias Físicas, Ciudad Universitaria s.n., Madrid 28040, Spain \\ *Corresponding author: jlvilas@ucm.es
}

Received 13 November 2012; revised 1 February 2013; accepted 4 February 2013; posted 5 February 2013 (Doc. ID 179853); published 14 March 2013

\begin{abstract}
Two plates of different birefringence material can be combined to obtain an achromatic wave retarder. In this work, we achieve a correction for the overall retardation of the system that extends the relation to any azimuth. Current techniques for the design of achromatic wave retarders do not present a parameter that characterizes its achromatism on a range of wavelengths. Thus, an achromatic degree has been introduced, in order to determine the optimal achromatic design composed with retarder plates for a spectrum of incident light. In particular, we have optimized a quarter retarder using two wave plates for the visible spectrum. Our technique has been compared to previous results, showing significant improvement. (C) 2013 Optical Society of America

OCIS codes: $\quad 220.4830,260.5430,260.1440,160.1190$.
\end{abstract}

\section{Introduction}

Circularly polarized light can be easily obtained with a linear polarizer and a quarter wave plate [1]. However, there exist applications where a high degree of circularity and stability is required, such as polarimetry, ellipsometry, optical activity, light polarization spectroscopy, optical communications cryptography, and photoelasticity $[1,2]$. In many applications, light is polychromatic. The use of achromatic circular polarizers on a region of the light spectrum has been studied for many years. One of the pioneers, Pancharatnam, proposed a configuration of three retarder plates, which produce an achromatic overall delay [3]. Hariharan achieved achromatic half-wave systems using retarders of quartz and mica [4]. Also, Gil and Bernabeu showed achromatic systems composed of two plates centered in a specific wavelength [5]. Hariharan and Malacara analyzed achromatic systems formed by two quarter-wave plates and one half-wave plate [6]. Boulbry et al. studied the errors associated with zero-order quarter wave plates [7].

$1559-128 \mathrm{X} / 13 / 091892-05 \$ 15.00 / 0$

(C) 2013 Optical Society of America
Recently, Saha et al. analyzed different methods to optimize achromatic systems composed of two retarder plates in several configurations $\lambda / 2+\lambda / 4$ and $\lambda / 4+\lambda / 4$ [8].

In this paper, we consider a system composed of two wave plates, and a novel expression for the overall retardation is calculated. The obtained expression is a generalization of the currently used equation to any azimuth [8-11]. In former works, achromatism is achieved only for two wavelengths and does not have a parameter that characterizes the performance of these achromatic designs. We present a new strategy for achromatism, optimizing a merit function that considers all the spectrum. By minimizing this merit function, which measures the distance between the retardation of the system in terms of the wavelength to target overall retardation, we can determine the thickness of the wave plates. We see that different materials for the two wave plates are required in order to obtain good achromatic systems. A numerical analysis is developed for different wavelength ranges. Our results are compared to those obtained by other authors, showing a significant improvement. In addition, this technique is extrapolable to other systems with more than two wave plates. 


\section{Theoretical Frame}

Let us consider a system formed by two wave plates, represented by their respective Jones matrices, $L_{1}$ and $L_{2}$, and characterized by the retardations $\delta_{1}$ and $\delta_{2}$, and the relative inclination of their fast axis with the horizontal, $\phi_{1}$ and $\phi_{2}$, respectively. This system, shown in Fig. 1, is illuminated with a polychromatic light beam with spectrum $g(\lambda)$. The notation of subindices used for all articles will be $i=1,2$, for the first and second plates. In this section we obtain the overall retardation of the system. Using Jones formalism, an ideal retarder plate, $L_{i}$, is defined by the following $2 \times 2$ matrix: of generality, $\phi_{1}=0$ and $\phi_{2}=\phi, \phi$ being the angle between the fast axes of the second plate and the first plate. Therefore the characteristic matrix of the system is the product

$$
\begin{aligned}
M= & L_{2} L_{1}=\left(\begin{array}{cc}
\cos \frac{\delta}{2}+i \sin \frac{\delta}{2} \cos 2 \phi & i \sin \frac{\delta}{2} \sin 2 \phi \\
i \sin \frac{\delta}{2} \sin 2 \phi & \cos \frac{\delta}{2}-i \sin \frac{\delta}{2} \cos 2 \phi
\end{array}\right) \\
& \times\left(\begin{array}{cc}
e^{i \delta_{1} / 2} & 0 \\
0 & e^{-i \delta_{1} / 2}
\end{array}\right)=\left(\begin{array}{cc}
A & B \\
-B^{*} & A^{*}
\end{array}\right),
\end{aligned}
$$

where $A=\exp \left(i \delta_{1} / 2\right)\left[\cos \left(\delta_{2} / 2\right)+i \sin \left(\delta_{2} / 2\right) \cos 2 \phi\right]$ and $B=i \exp \left(-i \delta_{1} / 2\right) \sin \left(\delta_{2} / 2\right) \sin 2 \phi$.

$$
L_{i}(\delta, \phi)=\left(\begin{array}{cc}
\cos (\delta / 2)+i \sin (\delta / 2) \cos 2 \phi & i \sin (\delta / 2) \sin 2 \phi \\
i \sin (\delta / 2) \sin 2 \phi & \cos (\delta / 2)-i \sin (\delta / 2) \cos 2 \phi
\end{array}\right)
$$

where the retardation, $\delta=2 \pi \Delta n(\lambda) d_{i} / \lambda$, depends on its thickness, the birefringence of the material $\Delta n(\lambda)=n_{e}(\lambda)-n_{0}(\lambda)$, and the wavelength. Refractive indices can be expressed using Sellmeier's equations [12]. $L_{i}(\delta, \phi)$ can be described in the basis of Pauli matrices, $\Sigma$, represented by four linearly independent matrices $2 \times 2, \Sigma=\left\{\sigma_{0}, \sigma_{1}, \sigma_{2}, \sigma_{3}\right\}$,

$$
\begin{aligned}
\sigma_{0}=\left(\begin{array}{ll}
1 & 0 \\
0 & 1
\end{array}\right), & \sigma_{1}=\left(\begin{array}{cc}
1 & 0 \\
0 & -1
\end{array}\right), \\
\sigma_{2}=\left(\begin{array}{ll}
0 & 1 \\
1 & 0
\end{array}\right), & \sigma_{3}=\left(\begin{array}{cc}
0 & -i \\
i & 0
\end{array}\right) .
\end{aligned}
$$

The set $\Sigma$ is complete and orthogonal, and Eq. (1) results in

$$
\begin{aligned}
L_{i}(\delta, \phi)= & \cos \frac{\delta}{2} \cdot \sigma_{0}+i \sin \frac{\delta}{2} \cos 2 \phi \cdot \sigma_{1} \\
& +i \sin \frac{\delta}{2} \sin 2 \phi \cdot \sigma_{2},
\end{aligned}
$$

where the $\sigma_{3}$ term does not appear. In order to simplify the expression, lets us consider, without loss

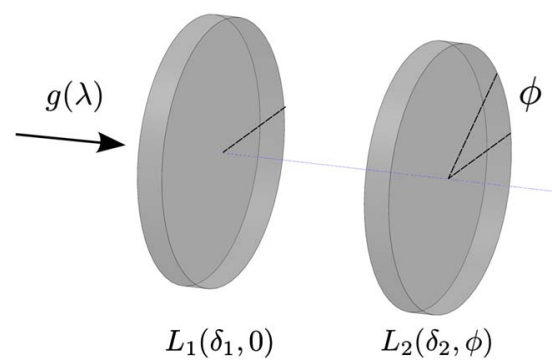

Fig. 1. (Color online) System to study. Two retarder plates $L_{1}$ and $L_{2}$ with retardations $\delta_{1}$ and $\delta_{2}$ and azimuths 0 and $\phi$, respectively, are illuminated with a polychromatic light beam with spectrum $g(\lambda)$.
Let us analyze the conditions under which the system, $M$, consisting of two plates, behaves as a single wave plate of azimuth $\Psi$ and gap $\Delta$. First, we decompose Eq. (4) in Pauli matrices

$$
M=l_{0} \sigma_{0}+l_{1} \sigma_{1}+l_{2} \sigma_{2}+l_{3} \sigma_{3},
$$

where

$$
\begin{aligned}
& l_{0}=\cos \frac{\delta_{1}}{2} \cos \frac{\delta_{2}}{2}-\sin \frac{\delta_{1}}{2} \sin \frac{\delta_{2}}{2} \cos 2 \phi, \\
& l_{1}=i\left(\cos \frac{\delta_{1}}{2} \sin \frac{\delta_{2}}{2} \cos 2 \phi+\sin \frac{\delta_{1}}{2} \cos \frac{\delta_{2}}{2}\right), \\
& l_{2}=i \cos \frac{\delta_{1}}{2} \sin \frac{\delta_{2}}{2} \sin 2 \phi, \\
& l_{3}=-i \sin \frac{\delta_{1}}{2} \sin \frac{\delta_{2}}{2} \sin 2 \phi .
\end{aligned}
$$

The equality $M\left(\delta_{1}, \delta_{2}, \phi\right)=L_{i}(\Delta, \Psi)$ implies that the component associated to $\sigma_{3}$ and $l_{3}$ must be zero, which only holds for $\phi=0$ or $\phi=\pi / 2$. Under this condition, equaling terms, we have that

$$
\tan ^{2} \frac{\Delta}{2}=\frac{|\operatorname{Im}(A)|^{2}+|\operatorname{Im}(B)|^{2}}{|\operatorname{Re}(A)|^{2}+|\operatorname{Re}(B)|^{2}}, \quad \Psi=0 .
$$

These expressions have been incorrectly used under the condition $\phi \neq 0[8,11]$. However, it is possible to calculate the overall delay, $\Delta$, introduced for any orientation of the plates, $\phi \neq 0$, via diagonalization of $M$. Since matrix $M$ is self-adjoint and unitary, the module of its eigenvalues, $\mu_{i}$, is unit

$$
\mu_{1}=s_{0}+\sqrt{s_{0}^{2}-1}, \quad \mu_{2}=s_{0}-\sqrt{s_{0}^{2}-1}
$$

where 


$$
s_{0}=\cos \frac{\delta_{1}}{2} \cos \frac{\delta_{2}}{2}-\sin \frac{\delta_{1}}{2} \sin \frac{\delta_{2}}{2} \cos 2 \phi .
$$

Consequently, the trace of the matrix product results in

$$
\operatorname{Tr}(M)=2 s_{0} .
$$

Finally, the overall retardation, $\Delta$, is obtained by equaling a diagonalized matrix with a diagonal matrix with eigenvalues $\mu_{1}=e^{i \Delta / 2}$ and $\mu_{2}=e^{-i \Delta / 2}$; that is,

$$
\left(\begin{array}{cc}
\mu_{1} & 0 \\
0 & \mu_{2}
\end{array}\right)=\left(\begin{array}{cc}
e^{i \Delta / 2} & 0 \\
0 & e^{-i \Delta / 2}
\end{array}\right),
$$

and, as a consequence,

$$
\cos \frac{\Delta}{2}=\frac{\operatorname{Tr} M}{2}=s_{0}
$$

Equation (11) can be used for an arbitrary orientation $\phi$ between the plates. Nevertheless, when $\phi=0$ or $\phi=\pi / 2$, there is an equivalence with Eq. ( $\underline{6})$.

\section{Achromatism}

Achromatic systems are commonly formed by two wave plates with different birefringence, and they present two configurations: in the first configuration, the fast axis of one of the wave plates is parallel to the slow axis of the other wave plate. In the second configuration, the fast axis of both wave plates is parallelly aligned $[4,7]$. Also, there are some works that use other azimuths $\phi$ [8]. In these works, achromatism is achieved with a contour condition applied to only two wavelengths without considering the spectrum of incident light. In addition, they do not have a parameter that characterizes the performance of these achromatic designs. The condition about the spectrum of incident light can be critical in order to optimize the behavior of achromatic retarders. For that reason, in the present work a merit function, achromatism degree (AcD), is defined in order to optimize the behavior in all the wavelength range

$$
\operatorname{AcD}=\frac{\left(\int\left|\Delta\left(\phi, d_{1}, d_{2}, \lambda\right)-\Delta_{0}\right|^{2} g(\lambda) \mathrm{d} \lambda\right)^{1 / 2}}{\int g(\lambda) \mathrm{d} \lambda},
$$

where $\Delta_{0}$ is the desired retardation and $g(\lambda)$ represents the spectrum of incident light. This merit function is a measure of the distance between the retardation of the system in terms of wavelength and a target overall retardation $\Delta_{0}$. This distance is weighted by the spectrum, in order to consider only those wavelengths with significant intensity. Therefore, the optimization of AcD will be adapted to the spectrum. Moreover, the AcD parameter represents a criterion in order to determine the achromaticity of optic systems. When the retardation obtained for all wavelengths is equal to the desired retardation $\Delta_{0}$, AcD is null. As this case is not fulfilled in the general case, when AcD presents a minimum, we will consider that we obtain the best achromatic result for the given spectrum.

In general, Eq. (12) cannot be solved analytically since, as shown in Eqs. (1) and (4), there is a complex relationship between $\Delta$ and the wavelength. Nevertheless, we can minimize $\mathrm{AcD}$ with respect to $\phi$, $\partial(\mathrm{AcD}) / \partial \phi=0$. For this minimization, there are two possible solutions for the azimuth, $\phi=0$ or $\phi=\pi / 2$, which are in agreement with the common experimental setups. Only under this result are Eqs. (11) and (6) equivalent. For the particular cases $\phi=0$ and $\bar{\phi}=\pi / 2$, Eq. (11) simplifies to

$$
\Delta=\delta_{1}+\delta_{2} \Leftrightarrow \phi=0, \quad \Delta=\delta_{1}-\delta_{2} \Leftrightarrow \phi=\pi / 2 .
$$

Since thickness cannot be negative, the minus sign in the second equation is related to an azimuth $\phi=\pi / 2$. As a consequence, both solutions are equivalent.

\section{Results}

After minimizing AcD with respect to $\phi$, we need to minimize with respect to the thickness of the wave plates $\left(d_{1}, d_{2}\right)$. The standard technique to obtain achromatic devices is making $\left|\Delta\left(\phi, d_{1}, d_{2}, \lambda\right)-\Delta_{0}\right|=0$ of Eq. (12) for just two wavelengths $\left(\lambda_{1}, \lambda_{2}\right)$, which are normally placed at the edges of the spectrum [4]. Then a system of two equations with two unknowns is obtained. For example, from Eq. (13), for a quarterwave retarder, this results in

$$
\begin{aligned}
& \Delta n_{1}\left(\lambda_{1}\right) d_{1} \pm \Delta n_{2}\left(\lambda_{1}\right) d_{2}=\lambda_{1} / 4, \\
& \Delta n_{1}\left(\lambda_{2}\right) d_{1} \pm \Delta n_{2}\left(\lambda_{2}\right) d_{2}=\lambda_{2} / 4 .
\end{aligned}
$$

Note that this system can be solved only when the birefringence of both plates is different. Therefore, the retarder plates must be manufactured with different materials.

A better solution can be obtained when the whole spectrum $g(\lambda)$ is considered for the minimization. Then, by numerical computations we can determine the thickness. As an example, let us consider an achromatic quarter-wave retarder, $\Delta_{0}=\pi / 2$, using two wave plates made of quartz and $\mathrm{MgF}_{2}$. These materials have been considered due to the abundant literature about them and are commonly used as retarder systems [, 13-18]. The ordinary, $n_{o}$, and extraordinary, $n_{e}$, refractive indices are defined by the Sellmeier's relations, which for $\mathrm{MgF}_{2}$ are

$$
\begin{aligned}
n_{o}^{2}-1= & \frac{0.48755108 \lambda^{2}}{\lambda^{2}-0.04338408^{2}}+\frac{0.39875031 \lambda^{2}}{\lambda^{2}-0.09461442^{2}} \\
& +\frac{2.3120353 \lambda^{2}}{\lambda^{2}-23.793604^{2}}, \\
n_{e}^{2}-1= & \frac{0.41344023 \lambda^{2}}{\lambda^{2}-0.03684262^{2}}+\frac{0.50497499 \lambda^{2}}{\lambda^{2}-0.09076162^{2}} \\
& +\frac{2.4904862 \lambda^{2}}{\lambda^{2}-23.771995^{2}},
\end{aligned}
$$

and for quartz, 


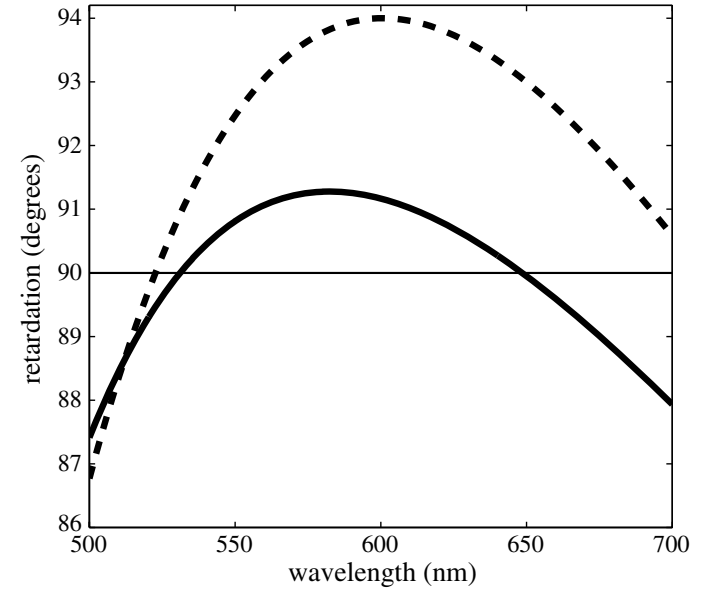

Fig. 2. Optimal overall retardation of the system for a Gaussian and plane spectrum (continuous curve) centered at $\lambda_{0}=600 \mathrm{~nm}$ and a width $\delta \lambda=100 \mathrm{~nm}$, with wavelength [500,700] nm. Both curves are coincident. A previous result of overall retardation is shown with a dashed curve [8].

$$
\begin{aligned}
n_{o}^{2}-1= & \frac{0.663044 \lambda^{2}}{\lambda^{2}-(0.0600)^{2}}+\frac{0.517852 \lambda^{2}}{\lambda^{2}-0.1060^{2}}+\frac{0.175912 \lambda^{2}}{\lambda^{2}-0.1190^{2}} \\
& +\frac{0.565380 \lambda^{2}}{\lambda^{2}-8.844^{2}}+\frac{1.675299 \lambda^{2}}{\lambda^{2}-20.742^{2}}, \\
n_{e}^{2}-1= & \frac{0.665721 \lambda^{2}}{\lambda^{2}-0.0600^{2}}+\frac{0.503511 \lambda^{2}}{\lambda^{2}-0.1060^{2}}+\frac{0.214792 \lambda^{2}}{\lambda^{2}-0.1190^{2}} \\
& +\frac{0.539173 \lambda^{2}}{\lambda^{2}-8.792^{2}}+\frac{1.8076613 \lambda^{2}}{\lambda^{2}-19.70^{2}} .
\end{aligned}
$$

With the definition of refractive indices given by the Sellmeier's relations, Eqs. (15) and (16), the AcD can be optimized in configurations of two retarder plates. For that reason, we applied Eq. (13) in (12), finding the thickness $d_{1}$ and $d_{2}$ such that Eq. (12) is minimal for a spectrum $g(\lambda)$. Since the integral Eq. (12) cannot be solved in the general case, a numerical calculus has been performed. This computation consists of determining AcD for each pair $\left(d_{1}, d_{2}\right)$ for an interval from 0 to $1000 \mu \mathrm{m}$ in steps of $1 \mu \mathrm{m}$. The best pair will be that whose parameter $\mathrm{AcD}$ is minimum. Sampling in steps of $1 \mu \mathrm{m}$ gives variation in the overall retardation less than or equal to $0.6^{\circ}$, which in consequence is a good approximation. As an example, the optimization will be performed in the wavelength interval $[500,700] \mathrm{nm}$ to achieve a quarter-wave plate.

We have considered two different light spectra: a Gaussian spectrum $g(\lambda)=\exp \left[-\left(\lambda-\lambda_{0}\right)^{2} /(2 \delta \lambda)\right]$ centered at $\lambda_{0}=600 \mathrm{~nm}$ and a width $\delta \lambda=100 \mathrm{~nm}$, and a plane spectrum, also centered at $\lambda_{0}=600 \mathrm{~nm}$ and a width $\delta \lambda=100 \mathrm{~nm}$. With the minimization of Eq. (12), we obtain the following thicknesses: $d_{1}=$ $371 \mu \mathrm{m}$ and $d_{2}=299 \mu \mathrm{m}$, for the both spectra. In Fig. 2 we can see the retardation produced by the system for the different wavelengths. The maximum difference, $\epsilon$, between the target retardation and the obtained, defined as

$$
\epsilon=\sup \left|\Delta-\Delta_{0}\right|
$$

results in $\epsilon=2.6^{\circ}$. The technique used by Saha to obtain an achromatic system produced a maximum difference $\epsilon=4^{\circ}$ for the same range of wavelengths [8].

Finally, let us see which is the maximum difference, $\epsilon$, for different spectrum bandwidths, using the spectra quoted, plane and Gaussian. We have considered the previous example for $\delta \lambda=25,50$, 75 , and $100 \mathrm{~nm}$. For each bandwidth, an optimization of Eq. (12) has been performed. In Fig. 3(a) we show the retardation curves $\Delta(\lambda)$ for these intervals. As the bandwidth intervals are smaller, the retardation curve is more plane. The maximum difference for the Gaussian or plane spectrum with the target retardation for the Gaussian or plane spectrum results in $\epsilon(50 \mathrm{~nm})=0.5^{\circ}, \epsilon(75 \mathrm{~nm})=1.2^{\circ}$, and $\epsilon(100 \mathrm{~nm})=$ $2.6^{\circ}$. For small intervals, the differences between (a)

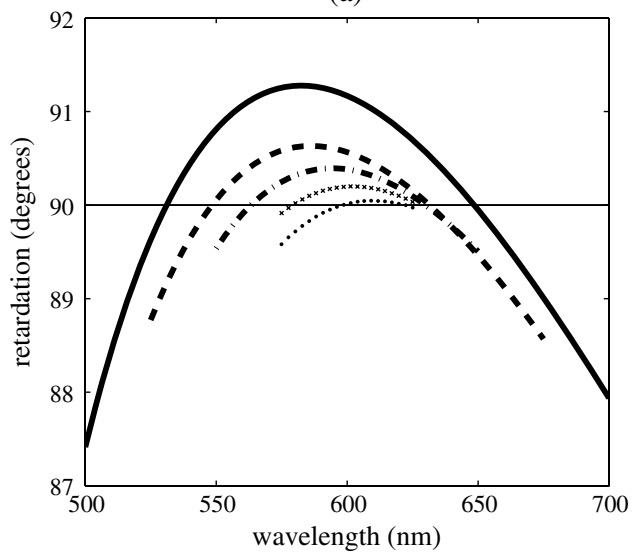

(b)

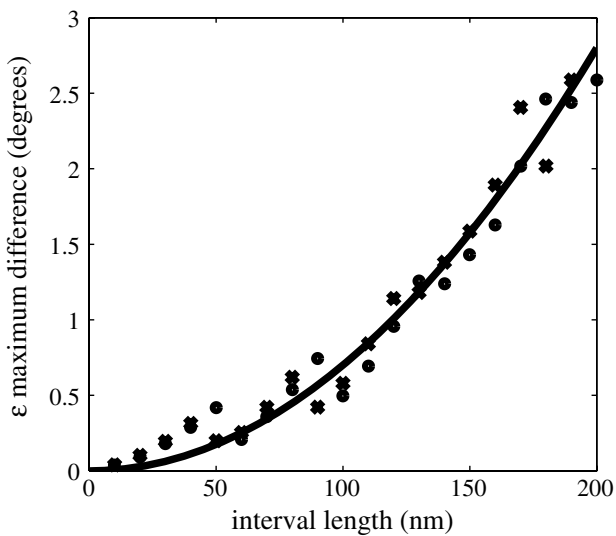

Fig. 3. (a) Overall retardation for plane or Gaussian spectra using several bandwidths: [500,700] nm (continuous), [525,675] nm (dashed), [550,650] nm (dash-dotted), and [575,625] nm (crosses for plane spectrum and dots for Gaussian spectrum). (b) Quadratic fit of maximum difference committed by the retarder as a function of the length interval for a Gaussian spectrum (dot points: continuous curve) and for a plane spectrum (cross points: dashed curve). Maximum difference, $\epsilon$, in terms of the bandwidth $\Delta \lambda$ for a plane spectrum (crosses) and a Gaussian spectrum (dots), and quadratic fit (continuous curve). 
both spectra increase. Indeed, when $\delta \lambda=25 \mathrm{~nm}$, the maximum difference for the plane spectrum is $\epsilon(75 \mathrm{~nm})=0.2^{\circ}$, and for the Gaussian spectrum is $\epsilon(75 \mathrm{~nm})=0.4^{\circ}$. Finally, in Fig. 3(b) we plot the maximum difference for a number of bandwidths for both spectra. These results have been fitted to a quadratic function, resulting $\epsilon(\delta \lambda)=7 \cdot(\delta \lambda)^{2}$, measured in degrees and nanometers. This equation is interesting since it allows us to adjust the bandwidth of the incident light in terms of the maximum difference with the target retardation.

\section{Conclusions}

In this work, the achromatism of a system formed by two wave plates has been studied. The overall retardation caused by the system has been determined via diagonalization of the matrix system. The equation obtained shows a generalization to any azimuth $\phi$, of the well-known Eq. (6), in which $\phi$ must be zero. We have defined a parameter, the $\mathrm{AcD}$, that accounts for the overall difference between the retardation produced by the system in terms of wavelength with respect to target retardation and the spectrum of incident light. This parameter give us a criterion for determining the achromaticity of the two wave plates. By minimizing this parameter, we show that the optimal azimuths must be $\phi=0$ and $\phi=\pi / 2$. Under these azimuths, instead of determining the thickness of the plates using only two wavelengths, a numerical minimization of the achromatic degree has been performed considering the whole spectrum. Much better results than previous works have been obtained. Finally, we show that the maximum retardation difference increases quadratically in terms of bandwidth.

This work was supported by the project "Photonic Transceiver for Secure Communications Space" of the European Space Agency with Tecnológica Ingeniería, Calidad y Ensayos, and by project DPI2011-27851 of the Ministerio de Ciencia e Innovacion of Spain.

\section{References}

1. D. S. Kliger, J. W. Lewis, and C. E. Randall, Polarized Light in Optics and Spectroscopy (Academic, 1990).

2. D. Goldstein, Polarized Light, 3rd ed. (CRC, 2003).

3. S. Pancharatnam, "Achromatic combinations of birefringent plates. Part II. An achromatic quarter-wave plate," Proc. Indian Acad. Sci. 41A, 137-144 (1955).

4. P. Hariharan, "Achromatic retarders using quartz and mica," Meas. Sci. Technol. 6, 1078-1079 (1995).

5. J. J. Gil and E. Bernabeu, "Diseño de rotores, compensadores y moduladores de retardo a partir de retardadores comerciales," Opt. Pura Apl. 15, 39-43 (1982).

6. P. Hariharan and D. Malacara, "A simple achromatic halfwave retarder," J. Mod. Opt. 41, 15-18 (1994).

7. B. Boulbry, B. Bousquet, B. Le Jeune, Y. Guern, and J. Lotrian, "Polarization errors associated with zero-order achromatic quarter-wave plates in the whole visible spectral range," Opt. Express 9, 225-235 (2001).

8. A. Saha, K. Bhattacharya, and A. K. Chakraborty, "Achromatic quarter-wave plate using crystalline quartz," Appl. Opt. 51, 1976-1980 (2012).

9. J. B. Masson and G. Gallot, "Terahertz achromatic quarterwave plate," Opt. Lett. 31, 265-267 (2006).

10. R. Pan, C. Lai, C. Lin, C. Hsieh, and C. Pan, "Achromatic liquid crystal phase plate for short laser pulses, molecular crystals and liquid crystals," Mol. Cryst. Liq. Cryst. 527, 65/[221]-71/ [227] (2010).

11. G. Kang, Q. Tan, X. Wang, and G. Jin, "Achromatic phase retarder applied to MWIR \& LWIR dual-band," Opt. Express 18, 1695-1703 (2010).

12. M. Born and E. Wolf, Principles of Optics: Electromagnetic Theory of Propagation, Interference and Diffraction of Light, 7th ed. (Cambridge University, 1999).

13. S. Chandrasekhat, "The dispersion and thermo-optic behavior of vitreous silica," Proc. Indian Acad. Sci. 34A, 275-282 (1951).

14. G. Ghosh, "Dispersion-equation coefficients for the refractive index and birefringence of calcite and quartz crystals," Opt. Commun. 163, 95-102 (1999).

15. J. M. Beckers, "Achromatic linear retarders," Appl. Opt. 10, 973-975 (1971)

16. M. Bass, C. DeCusatis, J. Enoch, V. Lakshminarayanan, G. Li, C. MacDonald, V. Mahajan, and E. Van Stryland, Handbook of Optics: Optical Properties of Materials, Nonlinear Optics, Quantum Optics, 3rd ed. (McGraw-Hill, 2009).

17. M. J. Dodge, "Refractive properties of magnesium fluoride," Appl. Opt. 23, 1980-1985 (1984).

18. P. D. Hale and G. W. Day, "Stability of birefringent linear retarders (wave plates)," Appl. Opt. 27, 5146-5153 (1988). 